\title{
Nanosonotechnology: the next challenge in cancer sonodynamic therapy
}

\section{Loredana Serpe*, Federica Foglietta and Roberto Canaparo}

Department of Drug Science and Technology, University of Torino, Via Pietro Giuria 13, Torino 10125, Italy, e-mail: loredana.serpe@unito.it

*Corresponding author

\begin{abstract}
Sonodynamic therapy (SDT) is a newly developed anticancer treatment where ultrasound is used to trigger the cytotoxic effect of chemical compounds, known as sonosensitizers. Although SDT is similar to photodynamic therapy (PDT), SDT activates the chemical compounds through energy transfer using ultrasound rather than light. Moreover, SDT can focus the ultrasound energy onto malignant sites situated deeply within tissues, thus overcoming the main drawback linked to the use of PDT. Several physical and chemical mechanisms underlying ultrasound bioeffects and anticancer SDT take advantage of the non-thermal effect of acoustic cavitation generated by selected pulsed or continuous ultrasound. As the physical-chemical structure of the sonosentizer is essential for the success of SDT, we believe that the different aspects related to nanotechnology in medicine might well be able to improve the triggering effect ultrasound has on sonosensitizing agents. Therefore, the aim of this review is to focus on how nanotechnology might improve this innovative anticancer therapeutic approach.
\end{abstract}

Keywords: cancer; nanoparticles; sonodynamic therapy; sonosensitizers; ultrasound.

\section{Introduction}

Ultrasonic waves are commonly described as waves that transport mechanical energy through the local vibration of particles without any net transport of the particles themselves. The term "ultrasound" is generally taken to mean that the frequency of the wave is greater than the upper threshold limit human hearing can detect. Therefore, ultrasound is a mechanical wave with periodic vibrations of particles in a continuous, elastic medium at frequencies $\geq 20 \mathrm{kHz}$ [1].

Nowadays, ultrasound is commonly used in most biomedical applications, both in the field of diagnosis and therapy, requiring better localization/focusing and higher frequencies (Table 1). However, even if ultrasound is generally considered "safe," as no harmful effects have been associated with their medical use, it can affect tissue through a variety of mechanisms, mainly thermal or non-thermal, depending on various factors such as the intensity of the beam and the duration of exposure and frequency (American Institute of Ultrasound in Medicine) [1].

There are several underlying mechanisms in ultrasound, i.e., streaming and radiation forces, but the most prominent mechanical effect on tissue is acoustic cavitation, which is defined as the formation or activity of gas- or vapor-filled cavities (bubbles) in a medium exposed to an ultrasound field [2].

Nanotechnology is defined as the study and use of structures with a size that varies between 1 and $100 \mathrm{~nm}$. The last few decades have witnessed an increase in the ability to manage nanosized materials, consequently, a world of possibilities in a variety of industries and scientific endeavors has been opened. As nanotechnology is essentially a set of techniques that allow for the manipulation of properties on a very small scale, it may have many and far-reaching applications. Indeed, one such important area is that of "nanomedicine," which, according to the National Institute of Health Nanomedicine Roadmap Initiative, refers to highly specific medical interventions at a molecular level for the diagnosis, prevention, and treatment of diseases [3]. Drug-delivery nanosystems make for a significant portion of nanomedicine. The importance of nanotechnology in drug delivery is in the concept and ability to manipulate molecules and supramolecular structures to produce devices with programmed functions. Clinically useful drug-delivery systems must be able to deliver a therapeutically effective amount of a drug, which is, more often than not, to be done over an extended period. The interaction of drug carrier systems with the biological environment is an important element on which to base and design strategies. These systems should be independent in the environment and selective at the pharmacological site. If appropriately designed, nanoparticles not only may act as a drug vehicle capable of targeting tumor tissues and/or cells but also offer a protective barrier against drug inactivation during transport. Therefore, the purpose of this review is to focus on how nanotechnology and, in particular, nanoparticles might improve the sonodynamic therapy (SDT) of cancer.

\section{Acoustic cavitation}

The non-thermal interaction of ultrasound with bulk liquid is accompanied by the quite unique phenomenon of cavitation, which leads to an enormous concentration and conversion of the diffused sound energy. Cavitation has at least 
Table 1 Frequency ranges and associated applications.

\begin{tabular}{ll}
\hline $16 \mathrm{~Hz}-20 \mathrm{kHz}$ & Human hearing \\
$20-100 \mathrm{kHz}$ & Conventional power ultrasound \\
$20 \mathrm{kHz}-3 \mathrm{MHz}$ & Sonochemistry \\
$1-3 \mathrm{MHz}$ & Physiotherapy \\
$3-30 \mathrm{MHz}$ & Diagnostic ultrasound \\
\hline
\end{tabular}

three discrete stages: nucleation, bubble growth and, under proper conditions, implosive collapse. The dynamics of cavity growth and collapse are strikingly dependent on the local environment; cavity collapse in a homogeneous liquid differs greatly from cavitation near an extended liquid solid interface [4, 5]. Cavitation has been classified into two types: non-inertial and inertial. Non-inertial cavitation bubbles oscillate about an equilibrium radius and often persist for many acoustic cycles. These oscillations create a streaming of surrounding liquid and mechanical stresses mix the medium. Inertial cavities are gas bubbles that grow to nearresonance size and may expand to a maximum, before collapsing violently (Figure 1). The temperature and pressure on the material contained within the imploding cavities can reach values $>10,000 \mathrm{~K}$ and $800 \mathrm{~atm}$. These extreme conditions may induce a multitude of chemical reactions (sonochemistry) both within and surrounding the bubble, including a concentration of energy that can generate light, an emission known as sonoluminescence [6].

Therefore, the wide range of application of acoustic cavitation also includes the direct interaction between the ultrasound and the biological membrane, modifying their permeability [7]. Indeed, ultrasound seems to be capable of modifying drug distribution in the human body, and it is hoped that a better understanding of such mechanisms will result in the optimization of drug therapies. Recently, numerous reports have been published on the application of ultrasound energy to target and/or control drug release in the fields of thrombolytic agents, transdermal drug delivery, anticancer drugs, and gene therapy [8]. Therefore, applying acoustic cavitation in an effort to increase the drug bioavailability is a very promising approach to drug-delivery systems and can be applied in different ways. First, ultrasound energy has the capacity to help agents penetrate through various tissues. Acoustic force is able to drive materials deeply into the skin, blood clots, or

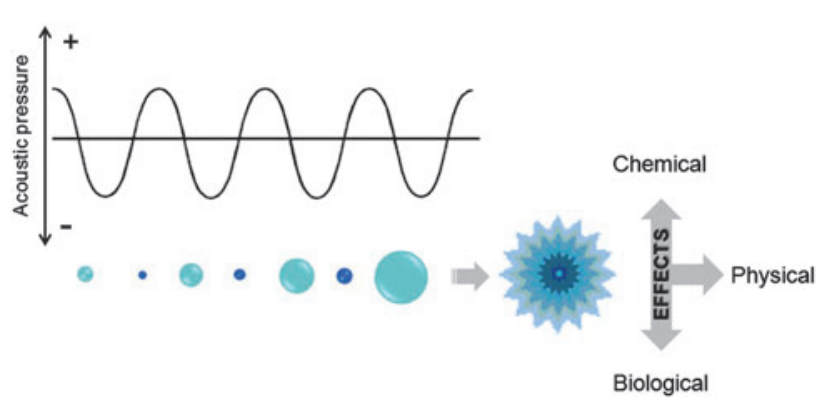

Figure 1 Drawing showing the formation, growth, and collapse of a cavitation bubble. other tissue. Researchers have hypothesized that these microjets, or the so-called microstreaming, could be applied to promote the diffusion of drugs into various tissues and lesions [7-9]. Indeed, acoustic energy exposure to specific cells by ultrasound, or shock waves, produced by lithotripters, have demonstrated an enhanced uptake of drugs within cells [8]. Another aspect is the use of the microbubbles to carry various drugs to target sites then rupturing the microbubbles by localized ultrasound energy. Indeed, albumin microbubbles were first used to further enhance the effects of thrombolytic agents when used in conjunction with ultrasound [10]. At moderately high sound pressure, amplitudes at the acoustic pressure waves can cause the rupturing of coated microbubble shells, allowing the bubbles to behave as non-coated microbubbles until they diffuse into the bloodstream. Moreover, drug-filled or drug-coated microspheres containing a therapeutic compound may be targeted to specific tissues through sonic energy that is directed to the target area and ruptures the microspheres, releasing the therapeutic compound. Even if the exact mechanism is still unknown, it is likely that the most exciting application of this method could well be gene therapy [11]. It is believed that once microspheres are ruptured, they can create a local increase in membrane fluidity, thereby enhancing the cellular uptake of the therapeutic compound [8]. Furthermore, targeted drug delivery assumes particular importance where drug toxicity is to be taken into careful consideration.

Ultrasound not only can enhance the drug bioavailability in vitro and in vivo, but they can also change the chemical property of the drug itself. It has been reported that light-sensitive materials, such as non-toxic agents such as hematoporphyrins, can be activated by ultrasound and may kill cancer cells [12]. Researchers discovered that non-thermal ultrasound may activate a group of chemicals that were originally used as light-activated chemicals for cancer therapy through acoustic cavitation, and this innovative ultrasound therapy has been termed "sonodynamic therapy" [8].

\section{Sonodynamic therapy}

SDT is one of the non-invasive therapeutic applications of ultrasound that has been considered in the last few decades [8, 13-15]. Although SDT is currently under investigation by researchers across the globe, this therapeutic approach is still in its early stage. Cytotoxicity is induced in SDT after the accumulation of a sonosensitizing agent in tumor cells by ultrasonic exposure through the activation of the sonosensitizer. As dedicated acoustic lenses can focus ultrasound into a limited focal zone, they are able to penetrate into the nonsuperficial tissues. The extent of the focal zone and penetration depth of ultrasound depend on both the focusing grade of the lens and the wave frequency [16].

Heat and mechanical effects are the most common bioeffects associated with ultrasound exposure and are both intensity and frequency dependent [14]. Therefore, the acoustic parameters for ultrasound therapy must be selected with care, taking the objective into consideration. The ultrasonic "level" may be determined by intensity, and Yu et al. [14] suggested 
$2 \mathrm{~W} / \mathrm{cm}^{2}$ as the critical value between low- and high-level ultrasound. Thus, ultrasound waves, which mostly induce structural alterations, have a high-level intensity, whereas those that mainly modulate tissue functions have a low level [14]. The thermal applications of ultrasound, such as high-intensity focused ultrasound (HIFU) [17], have been more extensively studied than has the therapeutic use of the non-thermal effects of ultrasound, even though it has been reported that free radical scavengers and antioxidants, such as mannitol, vitamins $\mathrm{C}$ and $\mathrm{E}$, and superoxide dismutase, could reduce cavitation-induced tissue damage [18].

One way to minimize heating is by using pulsed (e.g., shock waves), rather than continuous ultrasound [19, 20]. Generally, a high-energy shock wave (HESW) can be described as a single pulse with a wide frequency range (up to $20 \mathrm{MHz}$ ), highpressure amplitude (up to $120 \mathrm{MPa}$ ), low tensile wave (up to $10 \mathrm{MPa}$ ), small pulse width at $-6 \mathrm{~dB}$, and a short rise time $\left(<10 \mathrm{~ns}\right.$ ) (Figure 2). The energy density (up to $1.5 \mathrm{~mJ} / \mathrm{mm}^{2}$ ) and the pulse energy (up to $100 \mathrm{~mJ}$ ) determine the temporal and spatial distribution of the pressure profile. The energy density describes the maximum amount of acoustical energy, which is transmitted through an area of $1 \mathrm{~mm}^{2}$ per pulse [15]. Mechanical stresses seem to play a major role in high-energy shock waves, where the acoustic pressure, frequency of the applied ultrasound, pulse duration, and most importantly, the extent of nucleation of the exposed medium influence the occurrence of inertial cavitation. The likelihood of nucleation increases as the ultrasonic pressure amplitude increases.

As the sonosensitizers used in SDT have no or very low cytotoxicity without ultrasound stimulation, the cytotoxic effects of SDT only occur in the area where the localized ultrasound is applied (Figure 3). SDT, whether pulsed or continuous ultrasound, is considered to be safer and more readily accepted by the patient than either radiotherapy or chemotherapy, which often give rise to source concerns of safety and side effects [8]. SDT differs from photodynamic therapy (PDT), as drug activation is achieved through ultrasound rather than light. Moreover, SDT is able to overcome the main drawback of PDT, i.e., the relatively limited penetration of

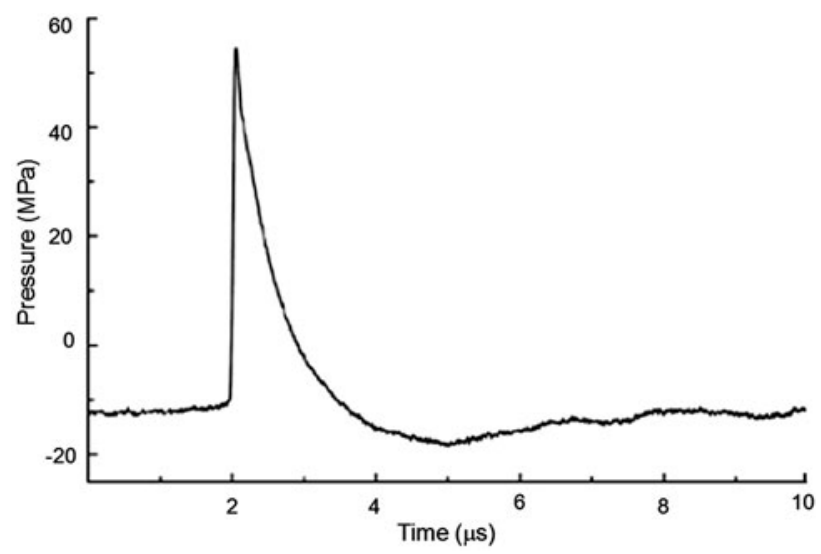

Figure 2 Pressure waveform of a shock wave as a function of time.

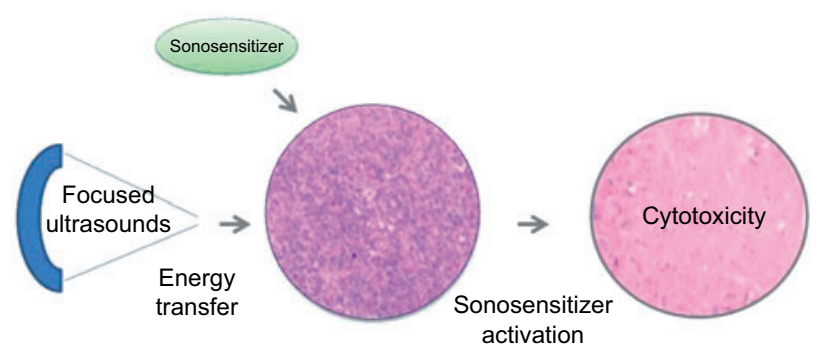

Figure 3 Anticancer SDT: cytotoxicity in tumor tissue when a sonosensitizer as been activated by focused ultrasound (hematoxylin and eosin staining of DHD/K12/TRb tumors photographed under light microscope at a $10 \times$ magnification [20]).

light into human tissue, and even at the longer wavelengths, light can penetrate no more than $5 \mathrm{~mm}$ into tissue. This goes without saying that this limits such treatments to surface (topical) applications, limiting PDT's application on superficial tumors, those that are directly underneath the skin surface, or on the surface of internal organs. Furthermore, in SDT, ultrasound may be used as an external remote control over time, dose, and location of drug release, providing tailor-made and localized cancer therapy [13].

A series of in vitro and in vivo trials confirmed that SDT, where either ultrasound or sonosensitizer had no or very low cytotoxicity, was efficacious in destroying malignant cells/tissues, and most sonosensitizers are either porphyrins or their derivatives (Table 2) [16, 21-27].

Protoporphyrin IX (PPIX) is an efficient hydrophobic tumor-localizing agent [28] that can be activated by both light and ultrasound [29]. The subsequent interaction of activated PPIX with molecular oxygen produces a cytotoxic reactive oxygen species (ROS), particularly singlet oxygen, which leads to an irreversible destruction of the target tissue. As the activation of the sonosensitizer is dependent on the cavitation process; high intensity ultrasound is a must. This followed the assumption that ultrasound energy might cause electronic excitation of porphyrins by energy transfer and initiate a photochemical process resulting in the formation of the cytotoxic singlet oxygen (Figure 4). Moreover, as opposed to anticancer drugs, porphyrins are non-toxic in the absence of ultrasound.

Unfortunately, some sonosensitizers have physical-chemical proprieties that seriously limit their clinical applications, such as poor water solubility. This is mainly true of natural products, such as hypocrellin, and nanotechnology is one method that can be adopted to solve such problems [27].

Table 2 Porphyrin-related sonosensitizers.

\begin{tabular}{llll}
\hline Sonosensitizers & Ref. & Sonosensitizers & Ref. \\
\hline ATX-70 & {$[21]$} & Photoprin II & {$[22]$} \\
ATX-S10 & {$[22]$} & Phthalocyanine & {$[25]$} \\
Chlorin-e6 & {$[23]$} & Protoporphirin IX & {$[24]$} \\
DCPH-P-Na & {$[22]$} & SF1 and SF2 & {$[26]$} \\
Hematoporphyrin & {$[24]$} & SL052 & {$[27]$} \\
\hline
\end{tabular}




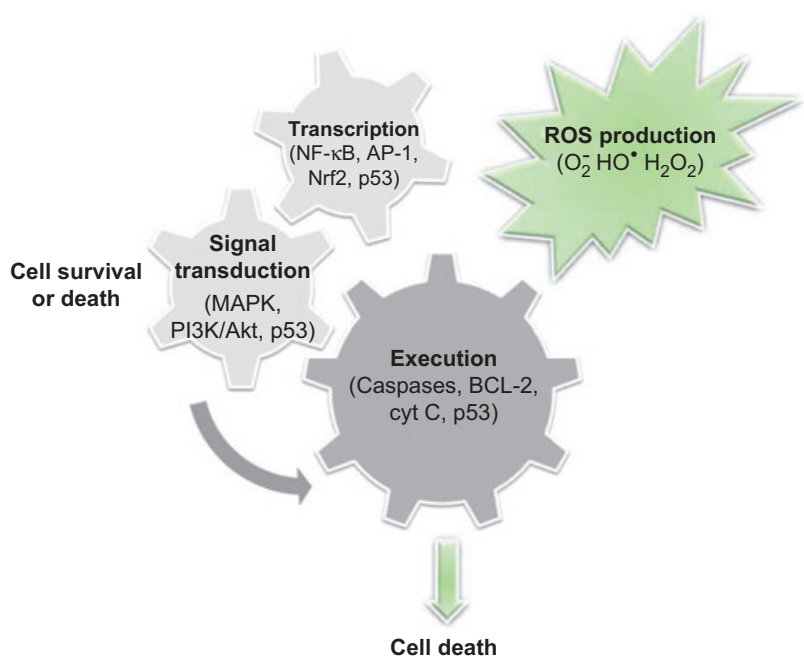

Figure 4 Redox-sensitive proteins that can regulate the cell fate decision through the regulation of signal transduction, transcriptional regulation, or execution. Not only does oxidative stress serve as a type of stimulus to trigger stress-response signal transduction pathways, but it can also modulate cell death/survival through direct oxidative modification of those signal molecules. ROS include radical species, such as superoxide $\left(\mathrm{O}_{2}^{-}\right)$and hydroxyl radical $\left(\mathrm{HO}^{*}\right)$ along with nonradical species, such as hydrogen peroxide $\left(\mathrm{H}_{2} \mathrm{O}_{2}\right)$.

Generally, nanoformulation plays an important role in improving drug delivery by making the drug more watersoluble without changing its physical-chemical structure. Furthermore, nanomodification allows the drug to be delivered to some deeply seated malignant sites that are not easily accessible to drugs. Therefore, we believe that nanotechnology approaches will improve SDT considerably.

\section{Nanoparticles as a sonosensitizer delivery system}

The tissue distribution of sonosensitizers is agent dependent and thus gives rise to several drawbacks in the bioavailability of sonosensitizing agents, as the therapeutic concentration of the chemical in a specific tissue may not be reached. That is to say, the concentration gradient between the malignant tissue and its adjacent normal tissue may not be sufficiently high to safely carry out SDT and the concentration in the target tissue might be lower than that in plasma, resulting in hemolysis and blood cell rupture. Therefore, the clinical application of today's sensitizers is not free from problems. Indeed, as most sonosensitizer molecules are hydrophobic and are consequently easily aggregated in aqueous media, the sonosensitizer aggregation decreases its efficacy for singlet oxygen production. Moreover, the aggregated sonosensitizer cannot be simply injected intravenously.

Yang et al. [30] describe an oral form of nanoparticles that encapsulate 5-aminolevulinic acid (5-ALA), a precursor of the sonosensitizer PPIX. Chitosan was mixed with sodium tripolyphosphate, and 5-ALA loaded chitosan nanoparticles (CNA) through the addition of 5-ALA with different $\mathrm{pH}$ values and concentrations. The results revealed that particle size with different $\zeta$-potential could be manipulated by the addition of 5-ALA with different concentrations and $\mathrm{pH}$ values. CNA particles, prepared at $\mathrm{pH} 7.4$ and $\mathrm{pH} 9$ of 5-ALA solutions at a concentration $>0.5 \mathrm{mg} / \mathrm{ml}$, showed a promising loading efficiency of up to $75 \%$ and an optimum average particle size of $100 \mathrm{~nm}$.

The triblock copolymer, Pluronic P105 (BASF, Mount Olive, NJ, USA), has been found to be an ideal ultrasonically activated drug-delivery vehicle [31]. The copolymer forms dense micelles with strongly hydrophobic cores at concentrations of $4 \mathrm{wt} \%$ or above and the polypropylene oxide core is sufficiently hydrophobic to sequester hydrophobic compounds. These micelles have been shown to release their contents upon the application of low-frequency ultrasound, suggesting that drug molecules can be delivered specifically to the insonated regions. Furthermore, once the ultrasound is turned off, the drug is quickly re-encapsulated inside the core of the micelles, preventing the spread of drug outside the targeted region [31]. The circulation time of the encapsulated drug is increased by polyethylene glycol (PEG) chains and prevents the carrier from being cleared by cells in the reticuloendothelial system. Indeed, pluronic micelles are large enough to escape renal excretion but small enough to extravasate at the tumor site. As pluronic has been shown to be non-toxic at low concentrations, it has been used to overcome multidrug resistance. Husseini et al. [32, 33] used an ultrasonic exposure chamber with real-time fluorescence detection to measure the release of doxorubicin from unstabilized and stabilized Pluronic P105 micelles through ultrasonic stimulation. Ultrasonic intensity increases the amount of drug release and frequency decreases it. Such ultrasonically controlled release has been effective against cancer cells both in vitro and in vivo $[31,34]$. It is noteworthy that the amount of release is not a linear function of ultrasonic intensity. In particular, there is a threshold at $0.3 \mathrm{~W} / \mathrm{cm}^{2}$. Observations of thresholds associated with cavitation and other ultrasonic phenomena are not unusual, and the existence of a threshold for the onset of inertial or collapse cavitation has been extensively documented [35, 36]. Other authors [1, 37] have calculated the threshold for the onset of inertial cavitation as a function of frequency and bubble size. Ultrasonic intensity thresholds reported for an observed biological effect $[1,11$, 35,38 ] usually differ from thresholds for collapse cavitation and vary according to the type of cell or tissue and the mechanism producing the effect (cell permeability, DNA delivery, cell lysis, or tissue damage) [31]. Husseini et al. [31] suggest that the increase in background acoustic emission, due to the wide spectrum of frequencies of shock waves (white noise), establishes the connection among collapse cavitation, the appearance of the subharmonic, and drug release from the micelles. Therefore, collapse cavitation may be involved in drug release, most probably through high shear forces that disrupt the structure of the micelle.

Drug delivery through nanoparticles is not the only approach that can enhance SDT (Figure 5). Indeed, the existence of a particle in a liquid provides a nucleation site for the cavitation bubble and, due to its surface roughness, leads 


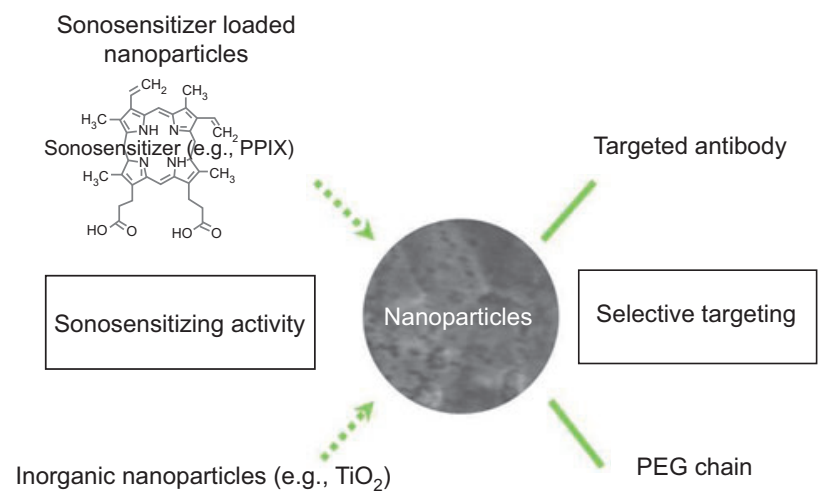

Nanosonosensitizers

Figure 5 Nanoparticles for sonosensitizing activity as sonosensitizer drug-delivery system and as nanosonosensitizers and for selective targeting as antibody-targeted and PEG-grafted nanoparticles.

to a decrease in the cavitation threshold responsible for the increase in the quantity of bubbles when the liquid is irradiated by ultrasound. Therefore, in this context, another nanotechnology approach might be based on providing the nucleation sites that participate in the formation of cavities to reduce the threshold intensity required for cavitation [39].

\section{Nanosonosensitizers}

The addition of particles to a liquid, in the appropriate size and amount, leads to an increase in cavitation bubbles and liquid temperature once ultrasound is applied. This suggests that the enhancement in the yield of sonochemical reaction by appropriate particle addition comes from an increase in the number of cavitation bubbles. Thus, the article of Tuziuti et al. [39] clarifies the fact that particle addition has the potential to enhance the yield of the sonochemical reactions, leading to an increasing interest in using nanoparticles as sonosensitizers. Moreover, nanomaterials have some features that make them particularly suitable for SDT from a pharmacological point of view, i.e., their being hydrophobic, having an enormous surface area that can be modified with functional groups that have a diverse array of chemical or biochemical properties, and a subcellular and submicrometer size that allows the nanoparticles to penetrate deeply into tissues with ease and be efficiently taken up by the cells (Figure 5). For example, gold nanoparticles are useful for SDT owing to their special optical properties. The low toxicity, good uptake by mammalian cells, and antiangiogenetic properties of gold nanoparticles make them highly attractive for medical applications [40]. Again, as inorganic nanoparticles often interact strongly with light, they can be used as therapeutic agents and diagnostic techniques for SDT. Indeed, research in this field has been carried out on the use of nanoparticle titanium dioxide $\left(\mathrm{TiO}_{2}\right)$ [41]. It is well known that, upon exposure to ultraviolet (UV) light, $\mathrm{TiO}_{2}$ particles can produce electrons, which lead to the formation of ROS, such as hydrogen peroxide, hydroxyl radicals, and superoxides. Furthermore, $\mathrm{TiO}_{2}$ was directly activated under the irradiation of UV and the potent toxicity of $\mathrm{TiO}_{2}$ has been combined with UV irradiation for use in melanoma C32 cells in vitro [41]. Moreover, it has been shown to inhibit tumor growth in a mouse xenograft model. However, the use of nanoparticle $\mathrm{TiO}_{2}$ still has some drawbacks, such as insufficient selectivity and low efficiency due to lack of cellspecific accumulation of the particles in cancer cells [41]. Xu et al. [42] reported that the combination of the electroporation and the conjugation of $\mathrm{TiO}_{2}$ with the monoclonal antibody could improve the photo-killing selectivity and efficiency of photo-excited $\mathrm{TiO}_{2}$ on cancer cells in PDT. Chihara et al. [43] found that liposome-encapsulated $\mathrm{TiO}_{2}$ might be more effective than non-coated $\mathrm{TiO}_{2}$ in the treatment of bladder cancer, and these modified $\mathrm{TiO}_{2}$ might also be a more effective material for SDT because ultrasound irradiation is an alternative energy source for $\mathrm{TiO}_{2}$ [43]. The recent clinical introduction of HIFU, the non-invasive thermal ablation method that can be targeted to tissues without damaging surrounding normal structures, could be applied as an alternative method to locally irradiating tumors in combination with $\mathrm{TiO}_{2}$ [41]. Yamaguchi et al. [44] hypothesized that sonocatalytic $\mathrm{TiO}_{2}$ might become a new tool in SDT for various cancers involving malignant glioma and developed a novel water-dispersed $\mathrm{TiO}_{2}$ nanoparticle, modified by PEG, demonstrating its photocatalytic antitumor effect on a glioma cell line. These nanoparticle compounds are approximately $50 \mathrm{~nm}$ in diameter and do not cross the blood-brain barrier (BBB) in the normal brain. On the contrary, the accumulation of the particles in malignant glioma by transvascular delivery can expected because the normal BBB is disturbed in the tumor site [45]. As the longterm side effects of inorganic nanoparticle accumulation in body organs are still unknown, luminescent silica nanoparticles that decompose in an aqueous solution over a period of hours have been developed [46]. Furthermore, Osminkina et al. [47] reported an in vitro anticancer effect of silicon nanocrystals used as a sonosensitizer.

\section{Complementary approach: nanoparticles for photoacoustic imaging}

Molecular imaging is a new biomedical research field that enables the visualization, characterization, and quantification of biological processes that take place at the cellular and subcellular levels within living tissues, including imaging modalities, such as that of photoacoustic and ultrasound imaging [48]. Molecular imaging always involves an accumulation of contrast agent in the target site; therefore, it has become evident that several of the challenges facing molecular imaging may be overcome by use of nanostructured materials such as nanoparticles. However, for physical reasons, nanoparticles have no role in ultrasound imaging because the frequencies necessary to detect particles smaller than $1000 \mathrm{~nm}$ diameter exceed $30 \mathrm{MHz}$, and when these are $>30 \mathrm{MHz}$, they do not penetrate into living tissues for more than a few millimeters. Furthermore, contrast media are based on air-liquid interfaces of high curvature (bubbles), and the creation of canonized bubbles is no easy feat. In ultrasound imaging, this 
gave evidence to the principle that molecular imaging can be achieved using microparticles (microbubbles, with diameters generally larger than about $5 \mu \mathrm{m}$ ) [49]. Ultrasound generated by optical stimulation of structures within a living tissue and nanoparticles may have important roles in "photoacoustic imaging" (PAI). Near-infrared optical imaging and PAI are both strong candidates when dealing with structures that are close to the surface, such as peripheral arteries and veins, which are of considerable interest in the screening of atherosclerosis [50]. PAI, sometimes called optoacoustic or thermoacoustic imaging, is a promising novel imaging modality for the non-invasive visualization of semitransparent objects, including soft biological tissues and biological samples. When short pulses of electromagnetic radiation, such as light or radio waves, illuminate a semitransparent object, a sound wave is induced at the point of interest deep within the sample via the thermoelastic effect [50]. The goal of PAI is the recovery of the spatially varying distribution of the absorbed energy inside the illuminated sample from the acoustic pressure measured outside the sample. As structures of different biological tissues reveal highly varying optical absorption coefficients, PAI makes a good distinction between malignant and healthy tissue.

Moreover, in the foreseeable future, it is expected to be useful in the early detection of cancer and blood-rich lesions in vivo and it is also expected to provide high resolution in microscopy [50]. Cancer detection using PAI with endogenous chromophores (e.g., hemoglobin and melanin) is an area of active research. One such report is on PAI that used to monitor melanoma tumor growth over the course of 2 weeks [51]. Optical contrast was provided by a higher concentration of melanin in the tumor compared with the surrounding tissue. PAI can also provide information on angiogenesis or vascular changes [52-54].

PAI systems have not only been used in the imaging of melanin and blood vessels but also in the measurement of blood oxygen content by comparing the photoacoustic signal strength of $\mathrm{HbO}_{2}$ and $\mathrm{Hb}$ obtained from spectroscopic photoacoustic images to study hypoxia in tumors, a negative prognostic factor often linked to therapy resistance $[55,56]$.

PAI has also been used to detect circulating tumor cells in the bloodstream, with the goal of detecting metastasis with contrast agents targeted to circulating tumor cells [57, 58]. Indeed, PAI has been shown to have the potential to identify, visualize, and track tumors and their associated vasculature with high resolution [59]. The sensitivity of the PAI technique to image deeply situated tumors can be further enhanced by exogenous contrast agents. Near-infrared-absorbing dyes, such as IRDye800CW [60], AlexaFluor 750 [61], and indocyanine green [62], have been used to enhance photoacoustic contrast.

However, among the various exogenous contrast agents, gold nanoparticles have attracted special attention due to their unique optical properties from the surface plasmon resonance effect, i.e., gold nanoparticles have an absorbance that is orders of magnitude higher than that of near-infrared dyes. For example, gold nanospheres, nanorods, nanoshells, nanocages, and nanobeacons have been used in PAI because of their tunable and strong longitudinal plasmon resonance in the near-infrared spectrum [59]. Specific molecular information regarding tumors can be obtained by attaching targeting moieties to exogenous agents. Gold nanoparticles with different optical absorption properties can be conjugated to cancer-specific biomarkers, such as growth factor receptors and integrins. Multiple targeted gold nanoparticles allow for multiplex molecular labeling of a tumor and multiwavelength PAI can evidence the heterogeneous accumulation and interaction of gold nanoparticles with cancer cells in vivo [59].

Recently, emphasis has been placed on multimodal nanosystems that can enhance contrast in two or more imaging modalities, including nanowontons consisting of a ferromagnetic (cobalt core) core coated with gold for biocompatibility and a unique shape that enables optical absorption over a broad range of frequencies. The magnetic core acts as a magnetic resonance imaging contrast agent and the gold coating provides the optical absorption contrast for the PAI [63]. The evolution of combined imaging strategies foresees a prominent role in cancer detection and treatment for multimodal nanostructures. Indeed, imaging techniques also have significant roles in cancer therapy, from precise planning and guiding to the evaluation of efficacy. PAI has shown a particular potential in therapies by providing a sequential monitoring of tumor functional properties, such as changes in the tumor vasculature before, during and after therapeutic procedures. Due to their high optical absorption properties in the near-infrared region, the therapeutic agents used for PDT can also act as photoacoustic contrast agents, e.g., PPIX for PDT [29] and gold nanoparticles for photothermal therapy [64].

PAI has an enormous potential in guiding therapeutic procedures and could well provide oncologists with structural and functional information on tumors to facilitate personalized therapy by customizing the therapy dose. Moreover, PAI can also be of help in customizing drug delivery with the use of multifunctional nanoparticles. An example of such a nanoagent is a silver nanosystem involving a poly(lacticco-glycolic acid) (PLGA) polymer core and outer silver cage network. The outer silver cage enhances contrast in PAI, and the inner core of the nanosystem contains the drug doxorubicin. Preliminary studies have indicated that the PLGA-based nanosystems have the potential to increase contrast significantly in PAI while simultaneously delivering customized payloads of drug to the tumor cells [65]. Another example of controlled and customized drug delivery is that of the use of light-triggered nanoconstructs, such as microspheres containing the drug paclitaxel encapsulated in hollow gold nanospheres. Depending on the concentration of gold nanospheres in the tumor, the light dose can be adjusted to allow for the correct release of paclitaxel [66].

\section{Summary and future directions}

Even though early results are promising [10, 27, 31, 44, 67, 68], perspectives are encouraging, and nanotechnology and SDT hold out the possibility of a cancer therapy, to date, nanosonotechnology is still in its early stage. Indeed, further 
research, both in vitro and in vivo, is required before it can be considered a clinical therapeutic tool against cancer. However, it has been demonstrated that nanoparticles can improve sonosensitizer bioavailability [30] and cancer targeting [69], opening new horizons in the development of a next generation of nanosonosensitizers, such as gold nanoparticles that carry PPIX [68]. As PPIX acts as a classical sonosensitizer and the gold nanoparticles increase the cavitation rate [70], it might be feasible to suppose that a synergistic effect on tumor cell killing is enhanced by the capability gold nanoparticles to increasing the amount of PPIX in the tumor cells. Indeed, in the presence of gold nanoparticles, the relaxation time of PPIX is longer than the time required for PPIX without gold nanoparticles [71] and favors an efficient generation of singlet oxygen.

Gold nanoparticle-PPIX conjugate has a greater potential than does PPIX alone as a sonosensitizer in the treatment of CT26 tumors by SDT. Such a finding can be related to a higher uptake of PPIX by the tumor cells through its nanoparticle carriers and the presence of gold nanoparticles as an enhancer of cavitation bubbles [67].

Furthermore, the new generation of PAI nanosonosensitizers might improve the therapeutic tools used against cancer. However, despite recent progress, optically active inorganic nanoparticles have not yet achieved broad clinical implementation, possibly due to the fact that drug loading is typically limited to the nanoparticle surface and there is still some concern about long-term safety, prompting further necessary investigation into the toxicity of inorganic nanoparticles $[64,72]$. Conversely, organic nanoparticles have found many human therapeutic applications due to their strong biocompatibility and drug-delivery capacity [73]. However, as these organic nanoparticles generally do not intrinsically absorb light in the near-infrared region, they have been of limited use for biophotonics. This led Lovell et al. [74] to introduce porphysomes, organic nanoparticles that are self-assembled from phospholipid-porphyrin conjugates and exhibit liposomelike structures and loading capacity, high absorption of nearinfrared light, structure-dependent fluorescence quenching, are enzymatically biodegradable and have an excellent biocompatibility. Porphysome are formed by supramolecular self-assembly, and the subunits consists of porphyrin-lipid conjugates generated by an acylation reaction between lysophosphatidylcholine and pyropheophorbide, a chlorophyllderived porphyrin analogue. This hydrophobic chromophore was positioned in place of an alkyl side chain, maintaining an amphipathic structure. A concentration of $5 \mathrm{~mol} \%$ PEGlipid was included in the formulation to enhance in vivo pharmacokinetics. Moreover, it has a stabilizing effect because PEG maintained their size and monodispersity for at least 9 months [75]. Porphysome are spherical vesicles measuring $100 \mathrm{~nm}$ in diameter, with two layers of higher-density material separated by a 2-nm gap, corresponding to two separate monolayers of porphyrin. They can form metal-chelating bilayers and are useful in a scenario where specific operating wavelengths are required. As porphysomes are higly self-quenched, energy that would normally be released to fluorescence and singlet-oxygen generation is dissipated elsewhere [74]. Porphysomes are intrinsically suited for multimodal imaging, such as photoacoustic tomography, and, together with other gold and magnetic nanoparticles, is promising approach in non-invasive detection of circulating cancer cells in blood vessels [76] as well as in sentinel lymph nodes [77]. In conclusion, it may be inferred that nanotechnology and SDT are two valuable approaches that can slow down the process of tumor growth, but the combination of "nanosonotechnology" might open an innovative and more effective scenario in cancer therapy.

\section{Acknowledgments}

The authors gratefully acknowledge funding from the Italian Ministry of Health and Piemonte Region (grant "Giovani Ricercatori 2008") and thank Barbara Wade for her advice on language.

\section{References}

[1] Leighton TG. What is ultrasound? Prog. Biophys. Mol. Biol. 2007, 93, 3-83.

[2] Maderbbacher S, Marberger M. High-energy shockwaves and extracorporeal high-intensity focused ultrasound. J. Endourol. 2003, 17, 667-672.

[3] Park K. Nanotechnology: what it can do for drug delivery. J. Control. Release 2007, 120, 1-3.

[4] Suslick KS. Sonochemistry. Science 1990, 247, 1439-1445.

[5] Suslick KS, Doktycz SJ, Flint EB. On the origin of sonoluminescence and sonochemistry Ultrasonics 1990, 28, 280-290.

[6] Didenko YT, Suslick KS. The energy efficiency of formation of photons, radicals and ions during single-bubble cavitation. Nature 2002, 418, 394-396.

[7] Tachibana K, Tachibana S. The use of ultrasound for drug delivery. Echocardiography 2001, 18, 323-328.

[8] Tachibana K, Feril Jr L, Ikeda-Dantsuji Y. Sonodynamic therapy. Ultrasonics 2008, 48, 253-259.

[9] Tachibana K, Tachibana S. Application of ultrasound energy as a new drug delivery system. Jpn. J. Appl. Phys. 1999, 38, 3014-3019.

[10] Siegel RJ, Atar S, Fishbein MC, Brasch AV, Peterson TM, Nagai T, Pal D, Nishioka T, Chae JS, Birnbaum Y, Zanelli C, Luo H. Noninvasive transcutaneous low frequency ultrasound enhances thrombolysis in peripheral and coronary arteries. Echocardiography 2001, 18, 247-257.

[11] Huber PE, Mann MJ, Melo LG, Ehsan A, Kong D, Zhang L, Rezvani M, Peschke P, Jolesz F, Dzau VJ, Hynynen K. Focused ultrasound (HIFU) induces localized enhancement of reporter gene expression in rabbit carotid artery. Gene Ther. 2003, 10, $1600-1607$.

[12] Umemura S, Yumita N, Nishigaki R, Umemura K. Mechanism of cell damage by ultrasound in combination with hematoporphyrin. Jpn. J. Cancer Res. 1990, 81, 962-966.

[13] Umemura S, Kawabata K, Sasaki K, Yumita N, Umemura K, Nishigaki R. Recent advances in sonodynamic approach to cancer therapy. Ultrason. Sonochem. 1996, 3, S187-S191.

[14] Yu T, Wang Z, Mason TJ. A review of research into the uses of low level ultrasound in cancer therapy. Ultrason. Sonochem. 2004, 11, 95-103.

[15] Shivastava SK, Kailash. Shock wave treatment in medicine. J. Biosci. 2005, 30, 269-275. 
[16] Rosenthal I, Sostaric JZ, Riesz P. Sonodynamic therapy - a review of the synergistic effects of drugs and ultrasound. Ultrason. Sonochem. 2004, 11, 349-363.

[17] Ahmed HU, Zacharakis E, Dudderidge T, Armitage JN, Scott R, Calleary J, Illing R, Kirkham A, Freeman A, Ogden C, Allen C, Emberton M. High-intensity-focused ultrasound in the treatment of primary prostate cancer: the first UK series. $B r . J$. Cancer 2009, 101, 19-26.

[18] Adinno MA, Al-Karmis AM, Stoltz PA, Matthews JC, Crum LA. Effect of free radical scavengers on changes in ion conductance during exposure to therapeutic ultrasound. $M e m b r$. Biochem. 1993, 10, 237-247.

[19] Canaparo R, Serpe L, Catalano MG, Bosco O, Zara GP, Berta L, Frairia R. High energy shock waves (HESW) for sonodynamic therapy: effects on HT-29 human colon cancer cells. Anticancer Res. 2006, 26, 3337-3342.

[20] Serpe L, Canaparo R, Berta L, Bargoni A, Zara GP, Frairia R. High energy shock waves and 5-aminolevulinic for sonodynamic therapy: effects in a syngeneic model of colon cancer. Technol. Cancer Res. Treat. 2011, 10, 85-93.

[21] Yumita N, Okuyama N, Sasaki K, Umemura S. Sonodynamic therapy on chemically induced mammary tumor: pharmacokinetics, tissue distribution and sonodynamically induced antitumor effect of gallium-porphyrin complex ATX-70. Cancer Chemother. Pharmacol. 2007, 60, 891-897.

[22] Kuroki M, Hachimine K, Abe H, Shibaguchi H, Kuroki M, Maekawa S, Yanagisawa J, Kinugasa T, Tanaka T, Yamashita Y. Sonodynamic therapy of cancer using novel sonosensitizers. Anticancer Res. 2007, 27, 3673-3677.

[23] Shi H, Liu Q, Qin X, Wang P, Wang X. Pharmacokinetic study of a novel sonosensitizer chlorin-e6 and its sonodynamic anticancer activity in hepatoma-22 tumor-bearing mice. Biopharm. Drug Dispos. 2011, 32, 319-332.

[24] Zhu B, Liu Q, Wang Y, Wang X, Wang P, Zhang L, Su S. Comparison of accumulation, subcellular location, and sonodynamic cytotoxicity between hematoporphyrin and protoporphyrin IX in L1210 cells. Chemotherapy 2010, 56, 403-410.

[25] Yumita N, Umemura S. Sonodynamic antitumour effect of chloroaluminum phthalocyanine tetrasulfonate on murine solid tumour. J. Pharm. Pharmacol. 2004, 56, 85-90.

[26] Lewis TJ. Toxicity and cytopathogenic properties toward human melanoma cells of activated cancer therapeutics in zebra fish. Integr. Cancer Ther. 2010, 9, 84-92.

[27] Meng Y, Zou C, Madiyalakan R, Woo T, Huang M, Yang X, Swanson E, Che J, Xing JZ. Water-soluble and biocompatible sono/photosensitizer nanoparticles for enhanced cancer therapy. Nanomedicine 2010, 5, 1559-1569.

[28] el Sharabasy MM, el Waseef AM, Hafez MM, Salim SA. Porphyrin metabolism in some malignant diseases. Br. J. Cancer 1992, 65, 409-412.

[29] Frank J, Lambert C, Biesalski HK, Thews O, Vaupel P, Kelleher DK. Intensified oxidative and nitrosative stress following combined ALA-based photodynamic therapy and local hypertermia in rat tumors. Int. J. Cancer 2003, 107, 941-948.

[30] Yang SJ, Shieh MJ, Lin FH, Lou PJ, Peng CL, Wei MF, Yao CJ, Lai PS, Young TH. Colorectal cancer cell detection by 5 -aminolaevulinic acid-loaded chitosan nanoparticles. Cancer Lett. 2009, 273, 210-220.

[31] Husseini GA, Diaz de la Rosa MA, Richardson ES, Christensen DA, Pitt WG. The Role of cavitation in acoustically activated drug delivery. J. Control. Release 2005, 107, 253-261.

[32] Husseini GA, Christensen DA, Rapoport NY, Pitt WG. Ultrasonic release of doxorubicin from Pluronic P105 micelles stabilized with an interpenetrating network of N,Ndiethylacrylamide. J. Control. Release 2002, 83, 302-304.

[33] Husseini GA, El-Fayoumi RI, O'Neill KL, Rapoport NY, Pitt WG. DNA damage induced by micellar delivered doxorubicin and ultrasound: comet assay study. Cancer Lett. 2000, 154, 211-216.

[34] Nelson JL, Roeder BL, Carmen JC, Roloff F, Pitt WG. Ultrasonically activated chemotherapeutic drug delivery in a rat model. Cancer Res. 2002, 62, 7280-7283.

[35] Hill CR. Ultrasonic Exposure Thresholds for Changes in Cells and Tissues. J. Acoust. Soc. Am. 1971, 52, 667-672.

[36] Kuijpers MWA, van Eck D, Kemmere MF, Keurentjes JTF. Cavitation-induced reactions in high pressure carbon dioxide. Science 2002, 298, 1969-1971.

[37] Flynn HG, Church CC. Transient pulsations of small gas bubbles in water. J. Acoust. Soc. Am. 1988, 84, 1863-1876.

[38] Barnett S. Thresholds for nonthermal bioeffects: theoretical and experimental basis for a threshold index. Ultrasound Med. Biol. 1998, 24, S41-S49.

[39] Tuziuti T, Yasui K, Sivakumar M, Iida Y, Miyoshi N. Correlation between cavitation noise and yield enhancement of sonochemical reaction by particle addition. J. Phys. Chem. A 2005, 109, 4869-4872.

[40] Ghosh P, Han G, De M, Kim CK, Rotello VM. Gold nanoparticles in delivery applications. Adv. Drug Deliv. Rev. 2008, 60, 1307-1315.

[41] Harada Y, Ogawa K, Irie Y, Endo H, Feril LB Jr, Uemura T, Tachibana K. Ultrasound activation of $\mathrm{TiO}_{2}$ in melanoma tumors. J. Control. Release 2011, 149, 190-195.

[42] Xu J, Sun Y, Huang J, Chen C, Liu G, Jiang Y, Zhao Y, Jiang Z. Photokilling cancer cells using highly cell-specific antibody$\mathrm{TiO}_{2}$ bioconjugates and electroporation. Bioelectrochemistry 2007, 71, 217-222.

[43] Chihara Y, Fujimoto K, Kondo H, Moriwaka Y, Sasahira T, Hirao Y, Kuniyasu H. Anti-tumor effects of liposomeencapsulated titanium dioxide in nude mice. Pathobiology 2007 , 74, 353-358.

[44] Yamaguchi S, Kobayashi H, Narita T, Kanehira K, Sonezaki S, Kudo N, Kubota Y, Terasaka S, Houkin K. Sonodynamic therapy using water-dispersed $\mathrm{TiO}_{2}$-polyethylene glycol compound on glioma cells: comparison of cytotoxic mechanism with photodynamic therapy. Ultrason. Sonochem. 2011, 18, 1197-1204.

[45] Sarin H, Kanevsky AS, Wu H, Brimacombe KR, Fung SH, Sousa AA, Auh S, Wilson CM, Sharma K, Aronova MA, Leapman RD, Griffiths GL, Hall MD. Effective transvascular delivery of nanoparticles across the blood-brain tumor barrier into malignant glioma cells. J. Transl. Med. 2008, 6, 80-94.

[46] Park JH, Gu L, von Maltzahn G, Ruoslahti E, Bhatia SN, Sailor MJ. Biodegradable luminescent porous silicon nanoparticles for in vivo applications. Nat. Mater. 2009, 8, 331-336.

[47] Osminkina LA, Gongalsky MB, Motuzuk AV, Timoshenko VY, Kudryavtsev AA. Silicon nanocrystals as photo- and sonosensitizers for biomedical applications. Appl. Phys. B 2011, 105, 665-668.

[48] Weissleder R. Molecular imaging in cancer. Science 2006, 26, $1168-1171$.

[49] Lindner JR. Evolving applications for contrast ultrasound. Am. J. Cardiol. 2002, 90, 72-80.

[50] Debbage P, Jaschke W. Molecular imaging with nanoparticles: giant roles for dwarf actors Histochem. Cell Biol. 2008, 130, 845-875. 
[51] Staley J, Grogan P, Samadi AK, Cui H, Cohen MS, Yang X. Growth of melanoma brain tumors monitored by photoacoustic microscopy. J. Biomed. Opt. 2010, 15, 40510-40513.

[52] Siphanto RI, Thumma KK, Kolkman RG, van Leeuwen TG, de Mul FF, van Neck JW, van Adrichem LN, Steenbergen W. Serial noninvasive photoacoustic imaging of neovascularization in tumor angiogenesis. Opt. Express 2005, 13, 89-95.

[53] Lao Y, Xing D, Yang S, Xiang L. Noninvasive photoacoustic imaging of the developing vasculature during early tumor growth. Phys. Med. Biol. 2008, 53, 4203-4212.

[54] Ku G, Wang X, Xie X, Stoica G, Wang LV. Imaging of tumor angiogenesis in rat brains in vivo by photoacoustic tomography. Appl. Opt. 2005, 44, 770-775.

[55] Lungu GF, Li ML, Xie X, Wang LV, Stoica G. In vivo imaging and characterization of hypoxia induced neovascularization and tumor invasion. Int. J. Oncol. 2007, 30, 45-54.

[56] Li ML, Oh JT, Xie XY, Ku G, Wang W, Li C, Lungu G, Stoica G, Wang LV. Simultaneous molecular and hypoxia imaging of brain tumors in vivo using spectroscopic photoacoustic tomography. IEEE Int. Ultrason. Symp. 2008, 96, 481-489.

[57] Zharov VP, Galanzha EI, Shashkov EV, Khlebtsov NG, Tuchin VV. In vivo photoacoustic flow cytometry for monitoring of circulating single cancer cells and contrast agents. Opt. Lett. 2006, 31, 3623-3625.

[58] Weight RM, Viator JA, Dale PS, Caldwell CW, Lisle AE. Photoacoustic detection of metastatic melanoma cells in the human circulatory system. Opt. Lett. 2006, 31, 2998-3000.

[59] Mallidi S, Luke GP, Emelianov S. Photoacoustic imaging in cancer detection, diagnosis, and treatment guidance. Trends Biotechnol. 2011, 29, 213-221.

[60] Louie A. Multimodality imaging probes: design and challenges. Chem. Rev. 2010, 110, 3146-3195.

[61] Razansky D, Vinegoni C, Ntziachristos V. Multispectral photoacoustic imaging of fluorochromes in small animals. Opt. Lett. 2007, 32, 2891-2893.

[62] Kim G, Huang SW, Day KC, O’Donnell M, Agayan RR, Day MA, Kopelman R, Ashkenazi S. Indocyanine-green-embedded PEBBLEs as a contrast agent for photoacoustic imaging. J. Biomed. Opt. 2007, 12, 044020.

[63] Bouchard LS, Anwar MS, Liu GL, Hann B, Xie ZH, Gray JW, Wang X, Pines A, Chen FF. Picomolar sensitivity MRI and photoacoustic imaging of cobalt nanoparticles. Proc. Natl. Acad. Sci. USA 2009, 106, 4085-4089.

[64] Boisselier E, Astruc D. Gold nanoparticles in nanomedicine: preparations, imaging, diagnostics, therapies and toxicity. Chem. Soc. Rev. 2009, 38, 1759-1782.

[65] Homan K, Shah J, Gomez S, Gensler H, Karpiouk A, BrannonPeppas L, Emelianov S. Silver nanosystems for photoacoustic imaging and image-guided therapy. J. Biomed. Opt. 2010, 15, 021316.

[66] You J, Shao R, Wei X, Gupta S, Li C. Near-infrared light triggers release of paclitaxel from biodegradable microspheres: photothermal effect and enhanced antitumor activity. Small 2010, 6, 1022-1031.

[67] Sazgarnia A, Shanei A, Meibodi NT, Eshghi H, Nassirli H. Anovel nanosonosensitizer for sonodynamic therapy: in vivo study on a colon tumor model. J. Ultrasound Med. 2011, 30, 1321-1329.

[68] Paciotti GF, Myer L, Weinreich D, Goia D, Pavel N, McLaughlin RE, Tamarkin L. Colloidal gold: a novel nanoparticle vector for tumor directed drug delivery. Drug Deliv. 2004, 11, 169-183.

[69] Yang SJ, Lin FH, Tsai KC, Wei MF, Tsai HM, Wong JM, Shieh MJ. Folic acid-conjugated chitosan nanoparticles enhanced protoporphyrin IX accumulation in colorectal cancer cells. Bioconjug. Chem. 2010, 21, 679-689.

[70] Wang S, Gao R, Zhou F, Selke M. Nanomaterials and singlet oxygen photosensitizers: potential applications in photodynamic therapy. J. Mater. Chem. 2004, 14, 487-493.

[71] Perez JLJ, Orea AC, Gallegos ER, Fuentes RG. Photoacoustic spectroscopy to determine in vitro the nonradiative relaxation time of porotoporphyrin IX solution containing gold metallic nanoparticles. Eur. Phys. J. Spec. Top. 2008, 152, 353-356.

[72] Lewinski N, Colvin V, Drezek R. Cytotoxicity of nanoparticles. Small 2008, 4, 26-49.

[73] Peer D, Karp JM, Hong S, Farokhzad OC, Margalit R, Langer R. Nanocarriers as an emerging platform for cancer therapy. Nat. Nanotechnol. 2007, 2, 751-760.

[74] Lovell JF, Jin CS, Huynh E, Jin H, Kim C, Rubinstein JL, Chan WC, Cao W, Wang LV, Zheng G. Porphysome nanovesicles generated by porphyrin bilayers for use as multimodal biophotonic contrast agent. Nat. Mater. 2011, 10, 324-332.

[75] Harris JM, Chess RB. Effect of pegylation on pharmaceuticals. Nat. Rev. Drug Discov. 2003, 2, 214-221.

[76] Galanzha EI, Shashkov EV, Kelly T, Kim JW, Yang L, Zharov VP. In vivo magnetic enrichment and multiplex photoacoustic detection of circulating tumour cells. Nat. Nanotechnol. 2009, 4, 855-860.

[77] Galanzha EI, Kokoska MS, Shashkov EV, Kim JW, Tuchin VV, Zharov VP. In vivo fibre-based multicolour photoacoustic detection and photothermal purging of metastasis in sentinel lymph nodes targeted by nanoparticles. J. Biophoton. 2009, 2, 528-539.

Received November 9, 2011; accepted January 18, 2012 


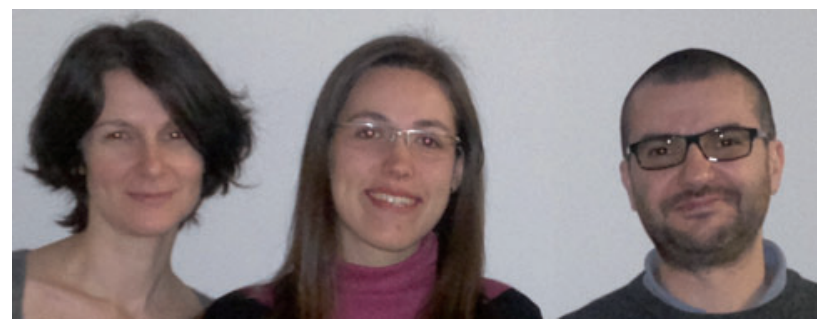

Loredana Serpe (left) was awarded her MD degree and $\mathrm{PhD}$ in Pharmacology and Toxicology at the University of Torino, Italy. She is currently working as a research fellow at the Department of Drug Science and Technology at the University of Torino. Her research includes nanoparticlebased drug delivery systems, sonodynamic therapy and pharmacogenetics-pharmacogenomics.
Federica Foglietta (center) is a $\mathrm{PhD}$ student in Pharmaceutical and Biomolecular Sciences at the Department of Drug Science and Technology at the University of Torino.

Roberto Canaparo (right) was awarded his $\mathrm{PhD}$ in Pharmacology and Toxicology at the University of Torino, Italy. He is a Lecturer in the Department of Drug Science and Technology at the University of Torino. He works in the field of nanoparticle-based drug delivery systems, sonodynamic therapy investigating the biological effects of shock waves, and in the field of pharmacogenetics-pharmacogenomics. 